\title{
Presencia, función y orden de constituyentes de algunas construcciones monovalentes en la Peregrinatio Egeriae
}

Concepción Cabrillana

(University of Santiago de Compostela)

\section{Presence, functionality, and constituent order of some monovalent structures in the Peregrinatio Egeriae}

\begin{abstract}
This paper has its starting point in the search for the causes of the very frequent presence of a possible monovalent predicate (fio) in the Peregrinatio Egeriae. The use of fio to present the development of repeated actions, often rituals, justifies its frequency - especially in the second part of the work - and, more specifically, the almost exclusive use of its passive factivity meaning. This fact is intrinsically linked to the use of the lexical or morphological passive to carry out this presentation of facts that usually constitute an entirely new piece of information from a pragmatic perspective, something that implies a determined order of constituents and the activation of an inversion mechanism, so that the majority sequence turns out to be Verb-S(ubject). This reality is corroborated by the study of the behaviour of predicates with similar characteristics and functionality. Among other related reasons, the selection of the passive - which has a pragmatic motivation and which is triggered as a textual cohesion device - to present the verbal process from the point of view of the event itself is revealed as one of the most explanatory causes of the concrete reality present in the text under investigation.
\end{abstract}

\section{Keywords}

monovalent structures; constituent order; fio; passive; Peregrinatio Egeriae; Verb-Subject inversion

Este trabajo se realiza en el marco (i) del Proyecto de Investigación FFI2017-83310-C3-2-P, denominado "Interacción del léxico y la sintaxis en griego y en latín: el predicativo y los procesos de copularización", financiado por el Ministerio de Economía y Competitividad del Gobierno español y por Fondos FEDER; (ii) del Proyecto "Compatibilidad de la Base de Datos REGLA con otros recursos digitales (COMREGLA)", financiado por una Ayuda de la Fundación BBVA a Equipos de Investigación Científica en las áreas de Economía y Sociedad Digital - Humanidades Digitales. 


\section{Justificación y estructura del estudio}

El análisis de una serie de datos pertinentes para el estudio de la evolución diacrónica en el proceso de copularización de varios predicados latinos ${ }^{1}$ arrojaba una serie de datos a primera vista sorprendentes para los que se veía necesario realizar un estudio particular. Más concretamente, se observaba que, en relación a otros textos de latín tardío fechados entre los ss. II y V d.C., la proporción de uso de uno de los predicados estudiados (fio) en la Peregrinatio era absoluta y propocionalmente muy superior a la que aparecía en el resto de textos, y de una uniformidad llamativa en cuanto a la noción semántica que denotaba.

Así pues, el objetivo original de este trabajo consistía básicamente en desentrañar los motivos y características de esa particular y concreta presencia de fio. Ahora bien, dado que la indagación para alcanzar ese objetivo se ha visto necesariamente ligada a una característica del tipo de estructuras objeto de estudio como es la del orden de sus constituyentes, ha sido preciso ampliar el objetivo inicial y abordar también la cuestión de las causas que podían explicar la ordenación relativa de los elementos obligatorios implicados en las estructuras que aparecían como prácticamente únicas con este verbo en la Peregrinatio. Este aspecto ha terminado exigiendo un tratamiento de tal peso que ha parecido más ajustado al contenido del trabajo incluirlo en el título para dar cumplida cuenta del conjunto de cuestiones investigadas. La estructura del trabajo sigue los siguientes pasos: en primer lugar se presentarán una serie de datos sobre el predicado en cuestión de manera comparativa con un conjunto de textos clásicos y tardíos (§2), unos datos de los que se extraerá una característica importante de este predicado, como es la de la ordenación de constituyentes en una de sus estructuras fundamentales. A continuación, se expondrán una serie de motivos que la investigación previa ha aducido para explicar este hecho de ordenación (\$ 3.1), y se procederá al análisis pormenorizado del comportamiento de fio y de otros verbos parcialmente similares en este aspecto ( $\S$ 3.2.1.-3.2.2) en el texto de la Peregrinatio. Por último, se resumirán las principales conclusiones que se pueden establecer tras el análisis realizado (§ 4).

\section{Los datos}

Como se sabe, el verbo latino fio es capaz de denotar diversas nociones semánticas, de las que las más frecuentes son la factivo-pasiva ("ser hecho"), la eventivo-existencial ("suceder") y la copulativa ("[llegar a] ser"); las dos primeras son monovalentes, mientras que la última es bivalente. ${ }^{2}$ También es conocida la dificultad existente en ocasiones para

1 Cabrillana (en prensa).

2 Una exposición y aplicación del modelo teórico seguido aquí y que defiende la relación entre el significado del verbo y las propiedades de los constituyentes considerados obligatorios que acompañan al predicado, puede verse, e.gr., en Dik (1989), Van Valin \& LaPolla (1997) o Pinkster (2015, 2021). 
identificar cuál es la noción concreta que se actualiza en cada aparición de las dos estructuras monovalentes posibles; ${ }^{3}$ sin embargo, la existencia de ambas está fuera de dudas. ${ }^{4}$

Uno de los datos llamativos afectaba, como se ha dicho, a la frecuencia de uso de este verbo frente a la utilización que hacían otros autores del corpus seleccionado entonces para realizar un estudio diacrónico sobre los procesos de copularización que sufrían los verbos fio, maneo, permaneo, remaneo, sto, exsto y exsisto. El corpus específico -variado en su tipología- estaba constituido por Apul. (met.), Tert. (apol., nat.), Peregr., Veg. (mulom.), Amm. (14-19), Symm. (epist. 1-5). La parcialidad de los textos de Amiano o Símaco se debía al intento de que hubiera la mayor proporcionalidad posible entre las cantidades de corpus a examinar, algo que no se conseguía de modo pleno, fundamental y precisamente por el menor volumen textual de la Peregrinatio; sin embargo, la representatividad de esta obra en los estudios evolutivos de la lengua latina por varios motivos y la singularidad de su tipo particular de texto aconsejaban no prescindir de su estudio. Los datos concretos se recogen en la siguiente tabla:

Tabla 1. Número de palabras en autores y obras del corpus de Latín tardío

\begin{tabular}{|l|c|}
\hline \multicolumn{1}{|c|}{ Autor - obra } & $\mathbf{N}^{\circ}$ de palabras \\
\hline Apul. (met.) & 53433 \\
\hline Tert. (apol., nat.) & $23529+15293=38822$ \\
\hline Peregr. & 17625 \\
\hline Veg. (mulom.) & 48409 \\
\hline Amm. (14-19) & $43589^{5}$ \\
\hline Symm. (epist. 1-5) & $38372^{6}$ \\
\hline TOTAL & $\mathbf{2 4 0 2 5 0}$ \\
\hline
\end{tabular}

Los ejemplos de fio en la totalidad de este corpus se reparten en la forma que refleja la siguiente tabla y, de manera gráfica y solo porcentualmente, el posterior gráfico:

Tabla 2. Distribución de ocurrencias de fio por autores-obras

\begin{tabular}{|c|c|c|c|c|c|c|}
\hline Verbo/Autor & Apul. & Tert. & Peregr. & Veg. & Amm. & Symm. \\
\hline fio (280x) & $9(3,21 \%)$ & $37(13,21 \%)$ & $134(47,85 \%)$ & $63(22,5 \%)$ & $11(3,92 \%)$ & $26(9,31 \%)$ \\
\hline
\end{tabular}

3 Cf. Cabrillana (2016, especialmente pp. 322-325) y la bibliografía citada ahí.

4 Cf. OLD, s.v. fio, 1-3, 12-13 (noción eventivo-existencial), frente a OLD, s.v. fio, 5-7, 11, 14-15 (noción factivo-pasiva).

5 Cifra aproximada, calculada sobre el total de las Res gestae (130768 palabras); puesto que solo se conservan 18 de los 31 libros que constituían la obra, en el cómputo se ha estimado lo que correspondería a la tercera parte de la misma (6 libros: 14-19).

6 Cifra de nuevo aproximada, calculada sobre la totalidad de las cartas (10 libros), que suma 76745 palabras. 
Presencia, función y orden de constituyentes de algunas construcciones monovalentes ...

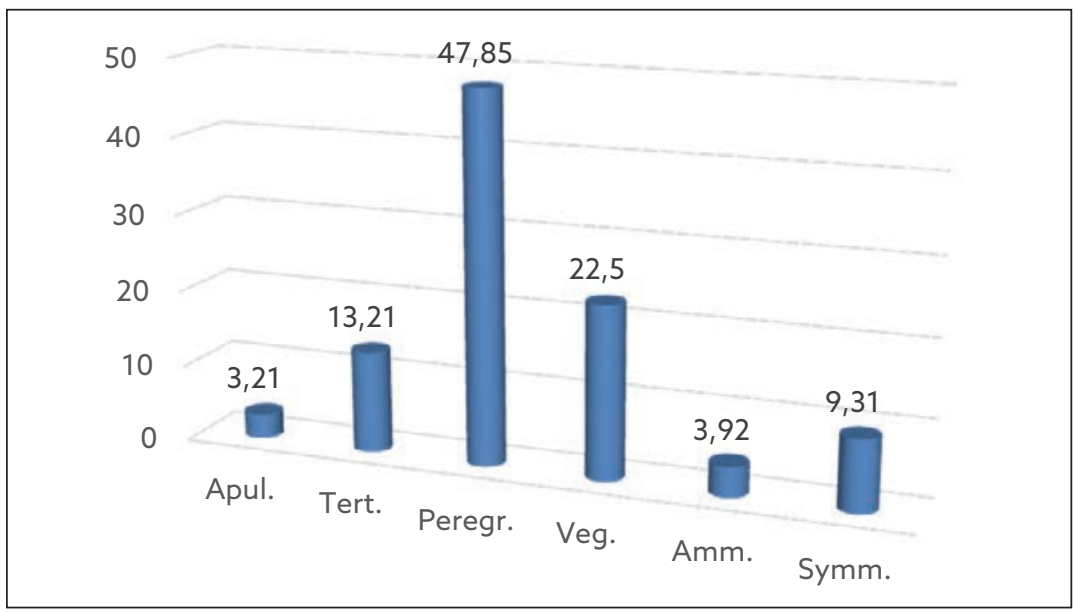

Gráfico 1. Porcentaje del empleo de fio por autores-obras

Como se ve, siendo la Peregrinatio el texto más corto de todo el corpus, exhibe un empleo de fio que incluso dobla el que realiza Vegecio, autor que suma casi tres veces el número de palabras de la obra de Egeria. Además de esta primera realidad -llamativa de por sí-, si se analiza la frecuencia de aparición de las distintas nociones del verbo aludidas más arriba en cada fuente textual, se obtienen los siguientes datos:

Tabla 3a. Distribución de ocurrencias de fio por acepciones más frecuentes y autores-obras: lectura "vertical"

\begin{tabular}{|l|l|l|l|l|l|l|}
\hline Valor semántico/Autor & Apul. & Tert. & Peregr. & Veg. & Amm. & Symm. \\
\hline $\begin{array}{l}\text { factivo-pasivo } \\
(164 \times=58,57 \%)\end{array}$ & $2(22,22 \%)$ & $11(29,72 \%)$ & $\begin{array}{l}131 \\
(\mathbf{9 7 , 6 6 \% )}\end{array}$ & $10(15,87 \%)$ & $8(72,72 \%)$ & $2(10,87 \%)$ \\
\hline $\begin{array}{l}\text { copulativo } \\
(66 \times=23,57 \%)\end{array}$ & $3(33,33 \%)$ & $16(56,74 \%)$ & $1(0,85 \%)$ & $40(36,50 \%)$ & 0 & $6(27,07 \%)$ \\
\hline $\begin{array}{l}\text { eventivo-existencial } \\
(50 \times=17,86 \%)\end{array}$ & $4(44,45 \%)$ & $10(27,02 \%)$ & $2(1,49 \%)$ & $13(20,63 \%)$ & $3(27,28 \%)$ & $18(62,06 \%)$ \\
\hline TOTAL (280x) & $\mathbf{9}$ & $\mathbf{3 7}$ & $\mathbf{1 3 4}$ & $\mathbf{6 3}$ & $\mathbf{1 1}$ & $\mathbf{2 6}$ \\
\hline
\end{tabular}

Tabla 3b. Distribución de ocurrencias de fio por acepciones más frecuentes y autores-obras: lectura "horizontal"

\begin{tabular}{|l|l|l|l|l|l|l|}
\hline Valor semántico/Autor & Apul. & Tert. & Peregr. & Veg. & Amm. & Symm. \\
\hline $\begin{array}{l}\text { factivo-pasivo } \\
(164 \times=58,57 \%)\end{array}$ & $2(1,22 \%)$ & $11(6,70 \%)$ & $131(79,88 \%)$ & $10(6,10 \%)$ & $8(4,88 \%)$ & $2(1,22 \%)$ \\
\hline $\begin{array}{l}\text { copulativo } \\
(66 \times=23,57 \%)\end{array}$ & $3(4,54 \%)$ & $16(24,24 \%)$ & $1(1,52 \%)$ & $40(60,61 \%)$ & 0 & $6(9,09 \%)$ \\
\hline $\begin{array}{l}\text { eventivo-existencial } \\
(50 \times=17,86 \%)\end{array}$ & $4(8 \%)$ & $10(20 \%)$ & $2(4 \%)$ & $13(26 \%)$ & $3(6 \%)$ & $18(36 \%)$ \\
\hline TOTAL (280x) & $\mathbf{9 ( 3 , 2 1 \% )}$ & $\mathbf{3 7 ( 1 3 , 2 1 \% )}$ & $\mathbf{1 3 4 ( 4 7 , 8 5 \% )}$ & $\mathbf{6 3 ( 2 2 , 5 \% )}$ & $\mathbf{1 1 ( 3 , 9 2 \% )}$ & $\mathbf{2 6}(\mathbf{9 , 3 1 \% )}$ \\
\hline
\end{tabular}


La lectura vertical de los datos - Tabla 3a- muestra más claramente la alta presencia del verbo analizado en la Peregrinatio y la abundantísima actualización de una misma acepción en esa obra, muy por encima de cualquiera de los demás autores. Por su parte, la lectura horizontal -Tabla 3b- ofrece como dato complementario cuál es la acepción de fio que más utiliza un autor determinado; así, la frecuencia en el empleo de cada una de las nociones semánticas principales de fio varía claramente de un tipo de texto a otro, algo ya apreciado, aunque con menor intensidad, en latín clásico. ${ }^{7}$ El estudio completo de estos resultados necesitaría de un trabajo independiente; lo que interesa resaltar aquí, de momento, es el abundantísimo uso de la significación factivo-pasiva del predicado en cuestión en el texto de Egeria: al tomar en consideración los diversos datos mostrados es inevitable preguntarse cuáles son las razones de ese comportamiento.

Ciertamente, en algunos estudios previos se ha llamado la atención sobre la frecuencia de fio en la Peregrinatio; así, Spevak (2005a: p. 237) lo presenta como el verbo más numeroso en oraciones principales $(98 \times)$ de la segunda parte de esta obra -donde se describen con mucha frecuencia eventos de distinto tipo-, frente a sum en la primera parte $(114 \times)$, esta de naturaleza más narrativa. La autora examina estos datos en un marco de análisis del orden de constituyentes en esta obra, donde constata (2005a: p. 257) una ordenación mayoritaria V(erbo)-S(ujeto) para los verbos monovalentes; posteriormente, Spevak (2010: p. 178) hablará también de una ordenación de fio en la que este aparece a la izquierda de la oración o al menos precede al S (en proporción de 8 ocurrencias sobre 10) en un corpus de latín clásico. ${ }^{8}$ La dificultad surge cuando se aprecia que estos datos, o bien provienen del conjunto de ocurrencias de fio, o bien se corresponden con los de su valor eventivo-existencial ("suceder - tener lugar"), que es el que parece otorgarle la autora cuando ilustra su afirmación con un ejemplo como el siguiente (2010: p. 178):

(1) fit celeriter concursus in praetorium ("a crowd quickly gathers in front of the commander's tent", ${ }^{9}$ Caes. civ. 1,76,2)

Con todo, los valores semánticos de los que habla Spevak son el de "devenir" (2005a: p. 237) y "to become” (2010: p. 174), que más bien se corresponderían con la noción copulativo-fientiva bivalente ("llegar a ser"). En este sentido, una investigación ${ }^{10}$ de la ordenación de constituyentes en construcciones monovalentes de fio sobre un corpus amplio de latín clásico, ${ }^{11}$ revelaba los siguientes datos:

$7 \quad$ Cf. Cabrillana (2016: pp. 315-318).

8 Caes. civ. 1-3,30; Cic. Tusc. 1 y 3, dom., Phil. 1 y 4, Att. 13,50-16; Sall. Iug.

9 Traducción ofrecida por Spevak.

10 Cabrillana (2017).

11 Catón (agr.), Plauto (Amph., Asin., Aul., Bach., Capt., Cas., Cucr., Epid.), César (Gall.), Salustio (Cat., Iug.), Cicerón (Verr., Catil., S. Rosc., Mil., Mur., Sest., dom., off.), Ovidio (met.), Livio 1-10, Columela, Plinio el Joven (pan.), Plinio el Viejo (nat. 1-15), Tácito (ann.), Séneca (dial. 6, 12, epist.), Petronio. 
Presencia, función y orden de constituyentes de algunas construcciones monovalentes ...

Tabla 4a. Ordenación de constituyentes según contenido semántico de construcciones monovalentes en latín clásico $(f i o)^{12}$

\begin{tabular}{|l|l|l|}
\hline Secuencia - Acepción & factivo-pasiva ("ser hecho") & eventivo-existencial ("suceder") \\
\hline SV & $196 \times(50,12 \%)$ & $47 \times(26,55 \%)$ \\
\hline S...V & $119 \times(30,43 \%)$ & $49 \times(27,68 \%)$ \\
\hline VS & $63 \times(16,11 \%)$ & $64 \times(36,15 \%)$ \\
\hline V...S & $13 \times(3,34 \%)$ & $17 \times(9,62 \%)$ \\
\hline Total S(...)V & $315 \times(80,56 \%)$ & $96 \times(54,23 \%)$ \\
\hline Total V(...) & $76 \times(19,44 \%)$ & $81 \times(45,77 \%)$ \\
\hline Total global: $568 \times$ & $391 \times$ & $177 \times$ \\
\hline
\end{tabular}

Si se analizan ahora los casos de la acepción mayoritaria en la Peregrinatio cuya ordenación no se encuentra condicionada por alguna constricción propia de la sintaxis latina $^{13}$ y en los que los dos elementos se encuentran explícitos, los resultados son los que aparecen en la tabla 4b:

Tabla 4b. Ordenación de constituyentes en acepción factivo-pasiva de fio en Peregr. ${ }^{14}$

\begin{tabular}{|l|l|}
\hline Secuencia - Acepción & factivo-pasiva ("ser hecho") \\
\hline SV & $9 \times(8,82 \%)$ \\
\hline S...V & $11 \times(10,78 \%)$ \\
\hline VS & $66 \times(64,70 \%)$ \\
\hline V...S & $16 \times(15,70 \%)$ \\
\hline Total S(...)V & $20 \times(19,41 \%)$ \\
\hline Total V(...)S & $83 \times(80,59 \%)$ \\
\hline Total global & $103 \times$ \\
\hline
\end{tabular}

Como es claro, la acepción factivo-pasiva muestra en la Peregrinatio una ordenación mayoritaria VS, contraria a la que aparece en latín clásico; este hecho se visualiza con claridad en el siguiente gráfico:

12 Cabrillana (2017: p. 434).

13 E.gr., la posición habitualmente inicial del pronombre relativo. Por otro lado, en este estudio, solo se analizan oraciones en modalidad declarativa.

14 Un estudio separado de oraciones principales y subordinadas (estas con mayor tendencia a presentar el verbo pospuesto al S y en posición final) apenas aporta variaciones, ya que las oraciones subordinadas constituyen únicamente 6 casos del total computado y solo en una ocasión aparece la secuencia SV. 


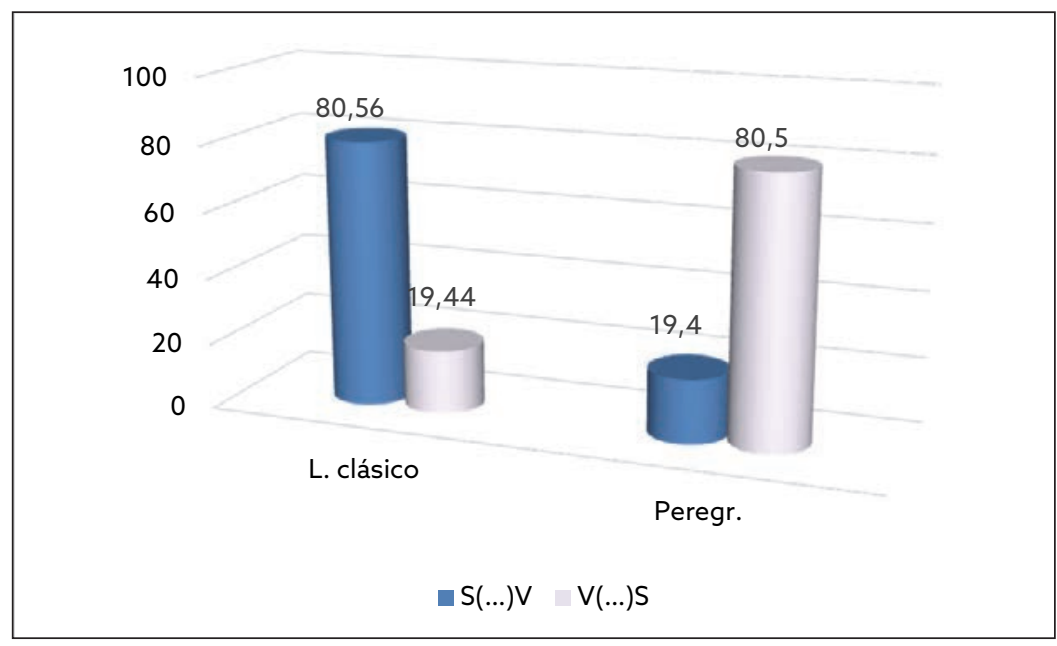

Gráfico 2. Porcentaje de las ordenaciones mayoritarias de fio factivo-pasivo en Latín clásico y en Peregr.

Esta disparidad con la situación en latín clásico justifica ya parcialmente la investigación propuesta aquí. Creo oportuno, además, ofrecer dos datos adicionales que ayudan a completar el panorama observable en la obra objeto de estudio:

(i) De un lado, la ordenación preponderante general de $\mathrm{O}$ (bjeto) y $\mathrm{V}$ en las estructuras con los tres elementos esenciales de las construcciones transitivas en la Peregrinatio resulta ser $\mathrm{V}(. ..) \mathrm{O}$; ahora bien, si se analiza la posición del $\mathrm{S}$ con respecto al $\mathrm{V}$ en este tipo de construcciones, se observa que las tendencias se invierten al comparar los datos generales de la obra con los correspondientes a la segunda parte de la misma; así, el cómputo de los datos en general arroja una secuencia preponderante S(...)V (66,2\%), mientras que en la segunda parte de la obra, el resultado mayoritario es $\mathrm{V}(\ldots) \mathrm{S}(60,3 \%) .{ }^{15}$

(ii) De otro, sería esperable que la ordenación de las secuencias donde aparecen formas pasivas de facio fuera paralela a la que exhibe fio. Sin embargo, del total de 35 apariciones analizables ${ }^{16}$ de facio factivo-pasivo, las preferencias resultan contrarias en cada una de las dos partes de la Peregrinatio: en la primera parte predomina V(...)S $(86,66 \%)$ y en la segunda $\mathrm{S}(\ldots) \mathrm{V}(85 \%) .{ }^{17}$

15 Porcentajes hallados a partir de los datos que ofrecen Hinojo (1986) y Pinkster (1991).

16 Esto es, cuando no existen constricciones que determinen la ordenación de constituyentes en un sentido u otro; p.e., el S aparece explícitamente y no está codificado por un pronombre relativo.

17 Entra dentro de lo probable que la mayor complejidad de una forma compuesta contribuya al retraso de la misma en la secuencia de la oración; se trataría, de algún modo, de una manifestación de la regla de complejidad creciente (Spevak 2010: p. 8). 
Estos datos hacen algo más complejo el cuadro que se dibuja en lo que respecta a la ordenación de constituyentes de la Peregrinatio y suponen, además, una nueva justificación del estudio que aquí se realiza.

\section{Causas de la inversión}

\subsection{Investigación previa}

El conjunto de los datos mostrados hasta aquí requiere, pues, un acercamiento y análisis pormenorizado del texto objeto de especial estudio.

De manera previa, sin embargo, resulta necesario repasar brevemente las explicaciones que se han proporcionado hasta ahora como justificaciones de la inversión (VS), ya sea en general o, mucho más concisamente, en relación al verbo fio.

Es algo relativamente admitido ${ }^{18}$ que los verbos intransitivos y monovalentes son más proclives a la ordenación VS, pero también se ha constatado que dichos verbos no constituyen sin más un grupo homogéneo. ${ }^{19}$ Teniendo esto en cuenta, el hecho de que se dé comienzo a una narración ${ }^{20}$ o descripción (eventualmente, junto a la presentación de circunstancias concomitantes) (2a) - quizá con el deseo de presentar el acontecimiento como algo inesperado $^{21}(2 b)$-, el que se trate de una oración presentativa ${ }^{22}$ (2c), el deseo de focalización de una propiedad o categoría verbal como la voz, el modo o el tiempo ${ }^{23}$ (2d-2e), o el que el verbo posea un valor especial de verdad ${ }^{24}(2 \mathrm{f})$ son otros tantos motivos que favorecen la posición inicial del verbo:

(2a) Mittitur ad eos colloquendi causa Gaius Arpineius, ..., et Quintus Iunius ex Hispania quidam, qui... ("Es enviado a tratar con ellos C. Arpineyo, ..., y cierto hispano llamado Q. Junio, que... ", ${ }^{25}$ Caes. Gall. 5,27)

(2b) Conclamatur ad arma ("Se da la voz de tomar las armas", Caes. civ. 1,69)

(2c) Erant in ea legione fortissimi uiri, centuriones, qui primis ordinibus appropinquarent, Titus Pullo et Lucius Vorenus. Hi... ("Había en esta legión dos centuriones valiosísimos, que pronto iban a ascender a los primeros órdenes, T. Pulón y L. Voreno. Estos...”, Caes. Gall. 5,44)

(2d) est idem Verres qui fuit semper ("es el mismo Verres que ha sido siempre”, Cic. Verr. 1,2)

18 Cf. Bolkestein (1995, 1996), Devine \& Stephens (2006: p. 151), Bauer (2009: pp. 280-281).

19 Cf. Spevak (2005b: p. 733).

20 Cf., entre otros, Devine \& Stephens (2006: pp. 158-159).

21 Cf. Luraghi (1995: pp. 367-373).

22 Cf. también, e.gr., Devine \& Stephens (2006: pp. 150-151).

23 Cf. Spevak (2004: pp. 382-383).

24 Cf. Bauer (2009: p. 278).

25 Si no se señala lo contrario, las traducciones de textos clásicos están basadas de las correspondientes a las de la Biblioteca Clásica Gredos, con esporádicas variaciones de matiz para recoger distintas características de las construcciones latinas, ausentes en esas fuentes. 
(2e) neque regerentur magis quam regerent casus ("(sc. los hombres) gobernarían al azar antes de ser gobernados por él”, Sall. Iug. 1,5)

(2f) Conatus est Caesar reficere pontes; sed nec magnitudo fluminis permittebat, ... ("César intentó realmente rehacer los puentes, pero ni lo permitía la magnitud de la crecida, ...”, Caes. civ. $1,50)$

Una ligazón interesante ha sido puesta de manifiesto por Bolkestein (1995) al advertir que la ordenación VS pueda estar vinculada a la presentación de una información nueva, ${ }^{26} \mathrm{o}$, en la terminología que utiliza la autora, "thetic": "where the state of affairs expressed is somehow presented as a whole - as a unified piece of information" (Bolkestein 1995: p. 32); ocurre en las presentativas y en las oraciones que responden a "¿qué ocurrió?", en oposición a “¿qué hizo X?":

(3) Reducitur ad eum deprensus ex itinere N. Magius Cremona, praefectus fabrum Cn. Pompei ("Es llevado a su presencia, hecho prisionero en el camino, Numerio Magio, de Cremona, prefecto de las tropas de ingenieros de Gneo Pompeyo", Caes. civ. 1,24)

La ordenación VS puede ser, pues, totalmente focal, pero también es posible que el foco esté constituido de una manera especial por el S (4a), y a veces no es fácil distinguir una posibilidad de la otra (Bolkestein 1995: pp. 39-40); a su vez, puede darse, asimismo, la secuencia SV para expresar una información totalmente nueva (4b) o focalizar el S (4c) (Bolkestein 1995: p. 41): ${ }^{27}$

(4a) prensat unus Galba ("solo hace campaña Galba", Cic. Att. 1,1,1)

(4b) tantus repente terror inuasit, ut... ("[Al conocerse en Roma estos acontecimientos,] se produjo de repente un terror tan grande que, ...”, Caes. civ. 1,14)

(4c) Eo L. Caesar adulescens uenit ("Llega allí Lucio César el joven”, Caes. civ. 1,8)

En realidad, esta autora (1995: pp. 32-33) va a mostrar que las condiciones que están presentes en la ordenación VS son heterogéneas -sintácticas, semánticas y pragmáticasy pueden superponerse e incluso parecer que en ocasiones entran en conflicto. ${ }^{28}$ Lo que sí ha demostrado Bolkestein (1996) para el latín clásico es la correlación existente entre el carácter pasivo del verbo y su posición a la izquierda de la oración, dado que con cuencia es el S el que proporciona una mayor aportación informacional, algo que facilita su posición postverbal.

26 Algo que constataba ya Väänänen (1987: p. 105).

27 Por otro lado, la escasa aparición de anafóricos, elementos de unión, conectivos, etc. en casos con ordenación VS ha llevado a Bolkestein a sugerir (1995: p. 43) que esta secuencia puede estar conectada con el hecho de que la visión del estado de cosas es 'separada' más que parte constituyente de una cadena continua.

28 En el mismo sentido y con una aplicación particular, cf. Cabrillana (2017). 
Tampoco ha pasado desapercibida la ordenación mayoritaria VS de estructuras monovalentes intransitivas en la Peregrinatio. ${ }^{29}$ No hay razón para pensar que los presupuestos semánticos y pragmáticos no son determinantes en el texto de Egeria en mayor o menor proporción.

En referencia más concreta a la posición de fio, Spevak (2010: p. 178) señala que este verbo poseería un contenido semántico débil y pocas posibilidades de ser foco; de modo similar a lo que señalaba Bolkestein (1995), Spevak piensa que las oraciones en las que aparece habitualmente este verbo responderían a la pregunta de “¿qué?”; 30 este hecho se corresponde con que en efecto, en la segunda parte de la Peregrinatio, haya una frecuente presencia de pasiva impersonal. ${ }^{31}$

Por otro lado, también se ha apuntado $^{32}$ para el texto de Egeria cierta correlación entre el carácter pasivo del verbo y su posición a la izquierda de la oración. Más concretamente, Spevak (2005a: pp. 253-254) señala que en la segunda parte de la Peregrinatio, los sujetos postpuestos responden en general a la necesidad de describir el desarrollo de acciones: ${ }^{33}$ "qué es lo que ocurre" y "quién está concernido por esa acción". Pues bien, la inactivación del agente que tiene lugar en la pasiva ${ }^{34}$ puede implicar que se presente el evento sin que se tenga que adoptar la perspectiva de participante alguno, y así, desde un punto de vista pragmático, convertirse en procedimiento para poner el acento en el proceso verbal denotado por el verbo. ${ }^{35}$ En tanto que la pasiva implica una conceptualización que no se orienta desde el agente, esta suerte de "inactivación", ${ }^{36}$ permite distintas posibilidades, de la que una es precisamente la presentación del proceso verbal sin hacer especial hincapié en quién lo realiza. Así, al presentar lo acontecido de manera concisa, se posibilita un significativo rendimiento literario.

Es este un punto que me parece de especial relevancia para explicar lo que de manera preponderante ocurre en la Peregrinatio. Así, si fio se considera como pasiva léxica de facio en su acepción factivo-pasiva, parecería lógico que habitualmente siguiera un esquema de ordenación VS cuando se utiliza con esta noción: al compartir en cierto modo el mecanismo de desagentivación de la pasiva, ese rasgo contribuiría a que el verbo se situase a la izquierda. ${ }^{37}$

Sin embargo, la comparación con lo que ocurre en época clásica (cf. Tablas 4a-4b, y Gráfico 2) plantea ciertos problemas. Ello puede deberse, además de a otras razones, a que en términos generales, en el discurso lingüístico y en el correspondiente procesado

29 Cf. Adams (1976: pp. 124-129), Väänänen (1987: pp. 104-105), Spevak (2005a: pp. 251-255), Ledgeway (2017: pp. 178-182).

30 Algo que la autora ejemplifica con (1).

31 Cf. Spevak (2005a: p. 254).

32 Cf. Väänänen (1987: p. 105), Spevak (2005a: p. 252). De modo más general, cf. Bolkestein (1995: p. 35; 1996).

33 Un análisis de textos de época clásica -Cabrillana (2017: pp. 432-433) - revelaba el uso más abundante de la acepción factivo-pasiva de fio en textos de tipo más realista y narrativo.

34 Cf. Haspelmath (1990).

35 Cf. Baños (2009: pp. 394-395).

36 Cf. Haspelmath (1997), Siewierska (2008).

37 Bolkestein (1995: pp. 35-36) ofrece datos claros que demuestran este hecho. 
de la información, un elemento limita al elemento que le precede desde el punto de vista informativo, lo que explica que el orden comunicativo habitual o no marcado sea Tópico-Foco, ${ }^{38}$ en congruencia con la denominada jerarquía de familiaridad ${ }^{39}$ «given > new» y la tendencia al dinamismo comunicativo creciente. ${ }^{40}$ Veamos pues, más en detalle, la realidad del texto tardío.

\subsection{Análisis particular de la Peregrinatio}

\subsubsection{El verbo fio}

Un examen pormenorizado de todos los casos de fio en la Peregrinatio revela la existencia de una gran cantidad de ocurrencias de expresiones a veces entendidas como cuasi-clichés ${ }^{41}$ que hacen referencia a un acto o rito que se lleva a cabo. Con todo, es importante matizar que este modo de ser "estereotipado" por parte de la pasiva del que habla Väänänen (1987: p. 62) habría de considerarse más bien un resultado de la frecuencia de uso sujeto a motivaciones pragmáticas y no tanto algo previo al mismo. En consecuencia, no han de entenderse este tipo de estructuras como construcciones concretas que están inicial y previamente ligadas de forma inexorable a un orden fijado, sino que en ellas intervienen también razones de orden semántico e informativo. ${ }^{42}$

Los dos ítems léxicos con los que este hecho se da de manera preponderante son oratio y missa. Este último término es susceptible de interpretarse básicamente de dos modos: Väänänen (1987: p. 32) señala que "le terme liturgique missa (72 ex. dans la seconde partie del l'Itin.) a les deux sens 'rite d'envoi' et 'office' en général" . ${ }^{43}$ En una relación de términos religiosos del texto estudiado, Väänänen (1987: p. 139) recoge lo siguiente: “missa (72), 1. 'le rite de renvoi' (38), 2. 'office' (avec ou sans eucharistie, celle-ci se nommant par ailleurs oblatio)". ${ }^{44}$ Por tanto, hay que entender que se trata con frecuencia de un acto en cierta medida ritual, con control por parte de quien lo lleva

38 Cf. Spevak (2010: pp. 28-29).

39 Este principio (Siewierska 1988: p. 61) explica también otras ordenaciones habituales: «more familiar > less familiar; definite > non definite; referential > non-referential», etc.

40 Sobre el concepto de "dinamismo comunicativo", cf. Firbas (1992: p. 7), Cabrillana (1992) y las matizaciones de Spevak (2008).

41 Algo constatado también en época clásica aunque sin tanta frecuencia (cf. Cabrillana 2017: pp. 449-450).

42 Cf. los frecuentes ejemplos justificados por una motivación pragmática que analiza Spevak (2005a).

43 Este autor (1987: p. 32) remite a lo que señala Maraval (1982: n. 3), que a su vez reenvía a Mohrmann (1958: pp. 67-92; esp. pp. 79 ss.). Gingras (1970: p. 217) alude asimismo a la consideración de missa como un término estilizado y casi cliché, que designaría mayoritariamente una serie de ritos poco definidos, constituyentes de las elaboradas despedidas características de las liturgias orientales; en algunos pasajes, sin embargo, el término haría referencia a un servicio religioso, como en Peregr. 24,6; 25,10; 27,8; 38,2 etc.

44 En un sentido similar, Wilkinson (1981: p. 57). En el ThLL, s.v., se lee: "missa fieri pronuntiatur"; 1136,7: "usu ecclesiastico (de notionibus non semper strictim discerni potest)"; 1136,12-13: "i. q. dimissio fidelium"; a continuación, dentro de ese sentido, el diccionario (s.v., 1136,11) recoge lo siguiente: "proprie: fit -a Peregr. Aeth. 24,2”, junto a bastantes citas más de esta obra; el texto concreto al que se hace referencia es el siguiente: et ille (sc. episcopus) eos uno et uno benedicet exiens iam, ac sic fit missa iam luce. 
a cabo, y que implica propiciar la despedida de los fieles; esta es la acepción mayoritaria, pero no exclusiva, ${ }^{45}$ en la Peregrinatio.

La naturaleza factivo-pasiva de estas expresiones queda clara al producirse la alternancia con su correlato formalmente pasivo:

(5a) Hoc autem lecto fit oratio ${ }^{46}$ ("Leído esto, se hace oración", ${ }^{47}$ Peregr. 37,8)

(5b) facta est ab episcopo oratio ("hizo oración el obispo", Peregr. 21,1)

(6a) et sic fit missa Anastasi (“y así se hace la despedida en la Anástasis”, Peregr. 24,6)

(6b) cum missa ecclesiae facta fuerit ("una vez que se ha hecho la despedida de la iglesia", Peregr. $25,10)$

Sin embargo, no parece seguro que se trate de auténticas colocaciones léxicas ${ }^{48}$ ni tampoco funcionales; ${ }^{49} \mathrm{el}$ ThLL (s.v. missa, 1137,36 ss.) recoge como iuncturae verbales las que se producen - se entiende que refiriéndose sobre todo al significado del rito eucarístico del término- con agere, cantare, celebrare, audire y comministrare; no aparecen, sin embargo, facere o fieri.

Un cómputo de la ordenación de las expresiones con estos ítems léxicos y otros menos frecuentes que comparten la acepción factivo-pasiva del verbo arroja los siguientes datos:

Tabla 5. Distribución de secuencias según algunos ítems léxicos $\mathrm{S}$ de fio con sentido factivo-pasivo ${ }^{50}$

\begin{tabular}{|l|c|c|}
\hline Ítem léxico/Ordenación & $\mathbf{S ( . . . ) V}$ & $\mathbf{V}(. ..) \mathbf{S}$ \\
\hline missa $(39 \times)$ & 6 & 33 \\
\hline oratio $(39 \times)$ & 2 & 37 \\
\hline oblatio $(4 \times)$ & 1 & 3 \\
\hline lucernare/lucernarium $(5 \times)$ & 1 & 4 \\
\hline uigilia $(3 \times)$ & 1 & 2 \\
\hline cathecisin $(1 \times)$ & 0 & 1 \\
\hline feria $(1 \times)$ & 1 & 0 \\
\hline predicatio $(1 \times)$ & 1 & 0 \\
\hline TOTAL $(93 \times)$ & $13(13,98 \%)$ & $80(86,02 \%)$ \\
\hline
\end{tabular}

45 Además de las cifras que porporciona Vaänänen (1987: pp. 32, 139), el ThLL, s.v. missa, 1136,68 incluye también: "strictius i. q. sacrificium sollemne Christianum, oblatio...", con cita, por ejemplo, de Peregr. 27,8 (Missa autem, quae fit sabbato ad Anastase, ante solem fit, hoc est oblatio, ...).

46 El texto latino de la Peregrinatio procede de la Base de datos de Brepolis (2001-2021).

47 Las traducciones de los textos de la Peregrinatio están tomadas de Arce (1980).

48 Definibles, de modo muy genérico, como "combinaciones restringidas de voces cuya frecuencia de coaparición es muy elevada” (Nueva gramática de la lengua española 2009: p. 2614); destacado tipográfico mío.

49 Es lo que también se denomina Construcción con Verbo Soporte; la Nueva Gramática de la Lengua Española (2009: p. 57), las define como "grupos verbales semilexicalizados de naturaleza perifrástica constituidos por un verbo y un sustantivo abstracto que lo complementa. Estos grupos admiten muchas veces... paráfrasis formadas con verbos relacionados morfológica o léxicamente con dicho sustantivo: dar un paseo ('pasear'), ..., etc. Estas paráfrasis son, sin embargo, aproximadas. De hecho, los verbos de apoyo suelen aportar contenidos aspectuales al significado expresado por el sustantivo que los complementa".

50 Se excluyen los casos de Sujetos pronominales que tienen estos ítems como referentes. 
La ordenación mayoritaria coincide con la tendencia ya observada en el cómputo general (Tabla 4b y Gráfico 2); ahora bien, creo que se ajustaría más a la verdad decir no tanto que la mencionada ordenación mayoritaria "sigue" esa tendencia, sino que, en realidad, constituye parte importante de su justificación. Es decir, la particular frecuencia de presentación o descripción del desarrollo de acciones ${ }^{51}$ que en conjunto suele ser información nueva y que con frecuencia recurre al uso de la pasiva, hace que la secuencia VS sea la preferida para la expresión de este tipo de situaciones. ${ }^{52}$

Si esta conclusión es correcta, cabría esperar que se diera el mismo comportamiento en otros verbos que en voz pasiva sean monovalentes y que compartan la característica de presentar o describir acciones: es lo que se aborda en el siguiente apartado.

\subsubsection{Otros verbos pasivos monovalentes}

Debemos a Spevak (2005a: p. 237) un listado de los diez verbos más fecuentes en cada una de las dos partes de la Peregrinatio, donde se aporta tanto el número total de ejemplos de esos verbos como la cantidad de ocasiones - cuando ello es posible- en que los distintos predicados aparecen en voz activa $(A)$ y pasiva $(P)$. Reproduzco a continuación dicho listado, añadiendo la marca "[]" en los casos en los que o bien no existe voz pasiva o su pasiva sería de tipo impersonal (itur, proceditur, etc.), de forma que no es posible la reconstrucción de un Sujeto; esta condición incapacita a esas formas para un análisis de construcciones monovalentes:

Tabla 6. Verbos más frecuentes en la Peregrinatio ${ }^{53}$

\begin{tabular}{|l|l|}
\hline Primera parte & Segunda parte \\
\hline$[114$ sum 'être' $]$ "* & 98 fio 'devenir' \\
\hline 34 ostendo 'montrer' $(16 \mathrm{~A}, 18 \mathrm{P})$ & 57 dico 'dire' (18 A, 39 P) \\
\hline 25 uideo 'voir' (19 A, 6 P) & {$[39$ sum 'être' $]$} \\
\hline 24 dico 'dire' (18 A, 6 P) & 31 ago 'faire' P \\
\hline 23 facio 'faire' (9 A, 14 P) & 29 lego 'lire' (6 A, 23 P) \\
\hline$[21$ peruenio 'arriver'] & 22 benedico 'bénir' (9 A, 13 P) \\
\hline$[14$ dignor 'vouloir bien'] & {$[18$ eo 'aller' P] } \\
\hline$[13$ habeo 'avoir'] & {$[16$ procedo 'aller' $(2 \mathrm{~A}, 14 \mathrm{P})]$} \\
\hline$[11$ coepi 'commencer'] & {$[12$ intro 'entrer'] } \\
\hline$[10$ aio 'dire'] & {$[12$ sedeo 'être assis' (10 A, 2 P) $]$} \\
\hline
\end{tabular}

[]*: casos no analizables

51 Especialmente en la segunda parte de la Peregrinatio: cf. Väänänen (1987: p. 61) o Spevak (2005a: pp. 253-254).

52 Spevak (2005a: p. 257) ya ofrecía el dato de que, para verbos monovalentes, en la segunda parte prima la secuencia VS (108 casos) frente a SV ( 45 casos).

53 Datos sobre los que proporciona Spevak (2005a: p. 237). 
Hecha esta salvedad obligada, veamos qué datos se obtienen de un análisis de los ejemplos de pasivas monovalentes en sus personas habituales y, con mucho, más frecuentes: $3^{\text {a }}$ singular y $3^{\text {a }}$ plural: ${ }^{54}$

Tabla 7. Distribución de secuencias en verbos monovalentes en voz pasiva en Peregr. ( $3^{\mathrm{a}} \mathrm{sg}$. y $3^{\mathrm{a}} \mathrm{pl}$.)

\begin{tabular}{|l|c|c|}
\hline Verbo/Ordenación & S(...)V & V(...)S \\
\hline dico $(59 \times)$ & 11 & 48 \\
\hline facio $(30 \times)$ & 13 & 17 \\
\hline ago $(20 \times)$ & 9 & 11 \\
\hline lego $(20 \times)$ & 3 & 17 \\
\hline benedico $(13 \times)$ & 0 & 13 \\
\hline ostendo $(4 \times)$ & 4 & 1 \\
\hline uideo $(4 \times)$ & 2 & 2 \\
\hline TOTAL $(151 \times)$ & $42(27,82 \%)$ & $109(72,18 \%)$ \\
\hline
\end{tabular}

En efecto, el resultado de esta indagación confirma la conclusión extraída del análisis de los datos de fio aunque en este caso la intensidad de las tendencias sea un tanto menos acentuada, algo que posiblemente obedece a una mayor variedad en los ítems léxicos con los que se combinan estos verbos, de modo que hay una menor probabilidad de cristalización de expresiones más o menos fijadas y, por tanto, un mayor margen de variación en la ordenación relativa de la secuencia resultante.

Los ejemplos son abundantes, y en no pocas ocasiones se observa cierta sucesión de construcciones similares, y que muestran características análogas:

(7a) In quo autem ingressus fuerit in anastase, dicitur unus ymnus, fit oratio, benedicuntur cathe-

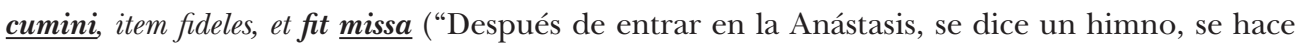
oración, son bendecidos los catecúmenos y los fieles, y se hace la despedida", Peregr. 32,2)

(7b) Vbi cum uentum fuerit, leguntur lectiones aptae, dicuntur psalmi uel antiphone, fit oratio, benedicuntur cathecumini et sic fideles, et fit $\underline{\text { missa }}$ ("Llegados allí, se leen las lecciones oportunas, se dicen salmos y antífonas, se hace oración, se da la bendición a los catecúmenos y a los fieles, y se hace la despedida", Peregr. 43,9)

54 Podría objetarse por qué no se incluye un verbo afín a los presentados como puede ser celebro: hay para ello un doble motivo. De un lado, he tomado como criterio de análisis el que ofrece Spevak sobre los diez verbos más frecuentes en ambas partes de la obra y celebro no figura entre ellos. De otro, el análisis de las ocurrencias del citado verbo con $3^{\mathrm{a}} \mathrm{sg}$. y $3^{\mathrm{a}} \mathrm{pl}$. arroja datos a primera vista contradictorios, puesto que de 10 casos en oraciones principales, 7 muestran ordenación SV. Sin embargo, un análisis más pormenorizado revela dos hechos importantes: (i) en la mayoría de los casos, esa ordenación viene en parte propiciada porque el $\mathrm{S}$ contiene un pronombre (is, hic, ipse, qui) de proyección anafórica y de condición topicalizada, que condiciona su anteposición, para hacer también más clara y cohesionada la narración o presentación de los hechos; (ii) en la única aparición con el item léxico missa como S, la ordenación es VS: Alia die autem, id est quinta feria quadragesimarum, celebratur missa ordine suo (Peregr. 42,1). 
De alguna manera, el estilo sin particular artificio en general de la Peregrinatio ${ }^{55}$ no evita la repetición de unas estructuras que, por otro lado, responden a razones justificadas.

Los siguientes textos recogen al menos un ejemplo de cada verbo analizado y que no aparece en las series de los dos ejemplos anteriores:

(8a) Et postmodum celebratis omnibus per ordinem, quae consuetudinis sunt, aguntur sacramenta et sic fit $\underline{\text { missa }}$ ("Y después de celebrarlo todo por orden, según la costumbre, se celebra la oblación, y luego se hace la despedida", Peregr. 26)

(8b) Denuo ergo et ad ipsum fontem, sicut et in singulis locis, facta est oratio et lecta est ipsa lectio ("Según la costumbre y práctica que teníamos al llegar a cualquiera de los lugares santos, también en esta fuente -como en cada lugar- se hizo oración, se leyó la lección”, Peregr. 15,4) (8c) ostenditur etiam ibi altarium lapideum ("muéstrase también allí un altar de piedra”, Peregr.

(8d) consuetudo est, ut fiat hic oratio ab his qui ueniunt, quando de eo loco primitus uidetur mons Dei ("Es costumbre que al llegar a este lugar, desde donde se ve por primera vez el monte de Dios, hagan aquí oración los que llegan”, Peregr. 1,7)

Las muestras de este tipo podrían multiplicarse. ${ }^{56}$

El espacio disponible para este trabajo impone pasar a las conclusiones finales. Hasta aquí, pues, el análisis esencial de los hechos y causas de la presencia de algunas construcciones monovalentes en el texto de la Peregrinatio, así como la determinación de su tipo, función y características pragmáticas y de ordenación de constituyentes asociadas.

\section{Conclusiones}

En esencia, puede decirse que la presencia particularmente abundante del verbo fio en la Peregrinatio se debe a su utilización para la presentación de hechos en un contexto básicamente narrativo donde de manera frecuente se da a concocer el desarrollo de acciones, a menudo rituales; en su gran mayoría, se trata de ocurrencias de la acepción factivo-pasiva de fio. En consonancia con ello, y con el propósito de poner ante el lector el hecho centrado en él mismo y no desde la perspectiva del agente, la lengua elige de

55 No es posible entrar aquí en esta debatida cuestión; cf., entre otros, Löfstedt (2007[1911]: pp. 8-20; 1959: esp. pp. 1-38) o Väänänen (1987: pp. 11-14) y la bibliografía recogida ahí. De modo sintético, podrían citarse las medidas palabras de Ernout (1954: p. 199) con relación especial al vocabulario de la obra: "Elle (sc. Egeria) a composé ce récit por faire participer à ce pélegrinage les soeurs de son abbaye (?) ..., et elle leur écrit dans un style simple, assez monotone, où abondent les formules, mais non parfois sans quelque prétention à la littérature".

56 Con todo, conviene insistir en que sería erróneo pensar que este modo de articular el discurso implique que la lengua de la Peregrinatio sea insensible o algo impermeable a la incidencia de factores pragmáticos: cf. Spevak (2005a: passim, p. 260); de hecho, es en buena medida el deseo de presentar la información como algo nuevo en su conjunto una de las causas que inciden en el modo de ordenar los constituyentes de las estructuras estudiadas aquí. 
manera preferente la voz pasiva para la expresión de este tipo de acontecimientos, que a menudo constituyen información nueva en su conjunto, algo que potencia la inversión de la secuencia habitual de los constituyentes, resultando así una ordenación mayoritaria VS. Si a ello se une además que las expresiones pasivas de verbos monovalentes tienden a esa ordenación VS, queda explicada y justificada la realidad presente en la Peregrinatio en torno a este tipo de construcciones. Entre otras razones concatenadas entre sí, parece, pues, que la selección de la pasiva -motivada pragmáticamente y por razones de cohesión textual- se revela como una de las causas principales para explicar la ordenación mayoritaria VS en las construcciones monovalentes analizadas.

De manera añadida, y como resultado de la presentación de hechos de cierta naturaleza ritual que se repiten, se observa el proceso de cristalización de algunas expresiones que facilita su consideración como secuencias más o menos fijas de constituyentes. ${ }^{57}$

\section{Bibliografía}

Adams, J. N. (1976). The text and language of a Vulgar latin chronicle: Anonymus Valesianus II (Bulletin of the Institute of Classical Studies of the University of London / Supplementary papers, 36). London: University of London, Institute of Classical Studies.

Arce, A. (Ed.). (1980). Itinerario de la Virgen Egeria (Biblioteca de autores cristianos, 416). Madrid: Ed. Católica.

Baños, J. M. (2009). Persona, número y voz. In J. M. Baños (Coord.), Sintaxis del latín clásico (pp. 375-403). Madrid: Liceus.

Bauer, B. L. M. (2009). Word order. In Ph. Baldi, \& P. Cuzzolin (Eds.), New Perspectives on Historical Latin Syntax, 1: Syntax of the Sentence (pp. 241-316). Berlin: Mouton de Gruyter.

Bolkestein, A. M. (1995). Functions of verb-subject order in Latin. Sprachtypologie und Universalienforschung - Language Typology and Universals, 48(1/2), 32-43.

Bolkestein, A. M. (1996). Free but non Arbitrary: 'Emotive' Word Order in Latin? In R. Risselada, J. R. de Jong, \& A. M. Bolkestein (Eds.), On Latin. Linguistic and literary studies in honour of Harm Pinkster (pp. 7-23). Amsterdam: Gieben.

Brepolis. (2001-2021). Brepols Publishers Online - Databases in the field of Humanities. [http:// clt.brepolis.net/llta/pages/QuickSearch.aspx; accessed 21.03.2021].

Cabrillana, C. (1992). Aproximación al concepto de 'Dinamismo Comunicativo'. Revista de la Sociedad Española de Lingüistica, 22(2), 397-404.

Cabrillana, C. (2016). Lexical domains in Latin predicative structures: 'agentive', fientive and stative types. In P. Pocetti (Ed.), Latinitatis rationes. Descriptive and Historical Accounts for the Latin Language (pp. 312-331). Berlin - Boston: De Gruyter.

57 Esta realidad se constataba también en textos de época clásica (Cabrillana 2017: pp. 449-450; Cabrillana 2020: pp. 17-24), en expresiones como fieri caedes / concursus / clamor / gemitus, etc. Sin embargo, existen también ejemplos de fio y de otros predicados analizados que muestran la ordenación SV con diversos ítems léxicos, por lo que hay que considerar este hecho con cierta prudencia. 
Cabrillana, C. (2017). Léxico, semántica y cognición en el orden de constituyentes latino. In J. de la Villa, E. Falque, J. F. González, \& Ma J. Muñoz (Eds.), Conuentus Classicorum. Temas y formas del Mundo Clásico (Vol. I; pp. 417-456). Madrid: Sociedad Española de Estudios Clásicos.

Cabrillana, C. (2020). Construcciones de fio + dativo: caracterización sintáctico-semántica. Euphrosyne, XLVIII, 9-26.

Cabrillana, C. (in print). Procesos de copularización en la lengua latina: precedentes semánticos y factores incidentes. „Glotta“.

Devine, A. M., \& Stephens, L. D. (2006). Latin Word Order. Structured Meaning and Information. New York - Oxford: Oxford University Press.

Dik, S. C. (1989). The Theory of Functional Grammar, I: The Structure of the Clause. Dordrecht: Foris. Ernout, A. (1954). Aspects du vocabulaire latin. Paris: Klincksieck.

Firbas, J. (1992). Functional sentence perspective in written and spoken communication. Cambridge: Cambridge University Press.

Gingras, G. E. (1970). Egeria: Diary of a pilgrimage. New York - Paramus: Newmann Press.

Halliday, M. A. K. (1994). An Introduction to Functional Grammar. London: Arnold (1 ${ }^{\text {st }}$ ed. London: Arnold 1985).

Haspelmath, M. (1990). The grammaticalization of passive morphology. Studies in Language, 14(1), 25-72.

Haspelmath, M. (1997). Indefinite Pronouns. Oxford: Oxford Clarendon Press.

Hinojo, G. (1986). El orden de palabras en la Peregrinatio Aetheriae. Studia Zamorensia Philologica, 7, 81-87.

Ledgeway, A. (2017). Late Latin Verb Second: The Sentential Word Order of the Itinerarium Egeriae. Catalan Journal of Linguistics, 16, 163-216.

Löfstedt, E. (2007 [1911]). Commento filologico alla Peregrinatio Aetheriae. Ricerche sulla storia della lingua latina (transl. P. Pieroni). Bologna: Pàtron.

Löfstedt, E. (1959). Late Latin. Oslo: H. Aschehoug.

Luraghi, S. (1995). The pragmatics of verb initial sentences in some ancient Indo-European languages. In P. Downing, \& M. Noonan (Eds.), Word Order in Discourse (pp. 355-386). Amsterdam - Philadelphia: Benjamins.

Maraval, P. (Ed. \& Transl.). (1982). Egérie. Journal de voyage (Itinéraire) (Sources Chrétiennes, 296). Paris: Éditions du Cerf.

Mohrmann, Ch. (1958). Missa. Vigiliae Christianae, 12(1), 67-92.

Nueva gramática de la lengua española (2009). Real Academia de la Lengua Española (Vol. II). Madrid: Espasa.

$O L D=$ Glare, P. G. W. (Ed.). (1982). Oxford Latin Dictionary. Oxford: Oxford University Press.

Pinkster, H. (1991). Evidence for SVO in Latin? In R. Wright (Ed.), Latin and the Romance Languages in the Early Middle Ages (pp. 69-82). London: Routledge.

Pinkster, H. (2015). The Oxford Latin Syntax, I: The Simple Clause. Oxford: Oxford University Press.

Pinkster, H. (2021). The Oxford Latin Syntax, II: The Complex Sentence and Discourse. Oxford: Oxford University Press.

Siewierska, A. (1988). Word Order Rules. Londres: Croom Helm.

Siewierska, A. (2008). Introduction: Impersonalization: An agent-based vs. a subject based perspective. Transactions of the Philological Society, 106(2), 115-137. 
Spevak, O. (2004). Verb-Subject order in Latin: the case of existential and locative sentences. Classica et Mediaevalia, 55, 381-386.

Spevak, O. (2005a). Itinerarium Egeriae: L'ordre des constituants obligatoires. Mnemosyne, 58(2), 235-261.

Spevak, O. (2005b). A propos de 'uerbum primo loco': essai de synthèse. In G. Calboli (Ed.), Latina Lingua! (pp. 731-740). Roma: Herder.

Spevak, O. (2008). The Position of Focus Constituents in Latin: A Comparison between Latin and Czech. Journal of Pragmatics, 40(1), 114-126.

Spevak, O. (2010). Constituent Order in Classical Latin Prose. Amsterdam - Philadelphia: Benjamins.

$T h L L=$ Thesaurus linguae Latinae editus auctoritate et consilio academicarum quinque Germanicarum Berolinensis Gottingensis Lipsiensis Monacensis Vindobonensis. (1900-). Leipzig - München - Berlin: Teubner; De Gruyter; Bayerische Akademie der Wissenschaften.

Van Valin, R. D., \& LaPolla, R. J. (1997). Syntax: structure, meaning and function. Cambridge: Cambridge University Press.

Väänänen, V. (1987). Le Journal-Épître d'Égerie (Itinerarium Egeriae). Étude linguistique. Helsinki: Suomalainen Tiedeakatemia.

Wilkinson, J. (1981). Egeria's travels to the Holy Land (rev. ed.). Jerusalem - Warminster: Ariel Publishing House; Aris \& Philips.

Prof. Concepción Cabrillana / concepcion.cabrillana@usc.es

Department of Classical, French and Italian Philology University of Santiago de Compostela, Faculty of Philology

Avda. de Castelao, s/n., 15782-Santiago de Compostela (La Coruña), Spain 